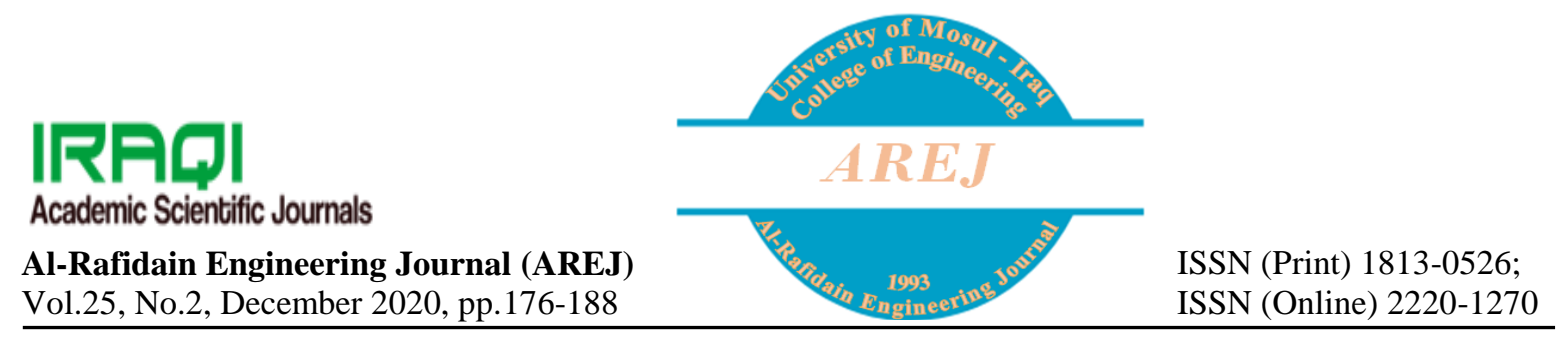

\title{
Simulation and Experimental Gait Cycle of Two Types of Degree of Freedom Bipedal Robot
}

\author{
Ali Fawzi AbdulKareem \\ a.abdulkareem0903@coeng.uobaghdad.edu.iq
}

\author{
Ahmed AbdulHussein Ali \\ ahmedrobot65@yahoo.com
}

Mechanical Engineering Departement, Collage of Engineering, University of Baghdad

Received: 7/7/2020

Accepted: $13 / 9 / 2020$

\begin{abstract}
Another type of legged robots is a bipedal walking robot or humanoid robot, which can be designed to implement various functions as necessary and mimic like a human. Often, balance while moving and when the first leg in the swing process and the second leg on the ground is difficult than most other kinds of robots. Two bipedal robot prototypes are designed with 10 degrees of freedom and 17 degrees of freedom to fulfill a gait cycle. The robot's locomotion can also be controlled via two types of microcontrollers, Arduino microcontroller and LOBOT LSC-32 driver. So, the KHR-2HV simulation model by Webots is used to simulate the experimental results of the bipedal robots. The results showed that the cubic polynomial foot trajectory for 10 degrees of freedom and 17 degrees of freedom bipedal robots are $(y=4 \times$ $10^{-16} z^{3}-0.0433 z^{2}+0.4329 z+0.7619$ with regression 0.9276$)$ and $\left(y=-0.000074 z^{3}-0.13 z^{2}+0.671 z+\right.$ 1.1326 with regression 0.939) respectively. After several methods for programming, the bipedal robot by LOBOT LSC-32 driver model is the better than Arduino with PCA 96685 driver-16 channel servo driver. Experimental results carried out during the KHR-2HV simulation model by Webots program. This model gives a better estimation and a fast response to confirm the stability of the 10 degrees of freedom and 17 degrees of freedom bipedal robots.
\end{abstract}

Keywords:

Degree of freedom; Gait robot; Simulation robot.

https://rengj.mosuljournals.com

Email: alrafidain_engjournall@uomosul.edu.iq

\section{INTRODUCTION}

Bipedal robot or humanoid is another type of robots and it has two legs. Besides, it is a significant replacement for the active robot because it can be moved on the unpaved land. Also, it is the most difficult than other types of robots to stability during walking or when the first leg in the swing phase and the second leg on the ground. First, a brief history of developing the design and control of the robot is studied.

Qiang Huang proposed an approach to study a foot trajectory and generate the foot trajectory through third-order periodic spline interpolation and a walking model consisting of a hip trajectory [1]. He found the hip trajectory by third-order periodic spline function and formulated the final hip trajectory with a large stability margin.
Shuuji Kajita et al. developed foot force controllers on the joint position servo and a body posture controller. By applying this force/posture control, the bipedal robot system as a simple linear inverted pendulum model (LIPM) with zero moment point (ZMP) delay is regarded [2]. They studied a tracking controller design for walking stabilization and sustained it by preliminary experiment. For more reliable and realize faster walk-in outdoor, the (LIPM) tracking controller must be improved.

Pandu Ranga Vundavilli and Dilip Kumar Pratihar focused on the stability of the gait creation problem for seven degrees of freedom (DoF) bipedal robot moving down and up through the sloping surface [3]. Sujan Warnakulasooriya et al. investigated the design of a humanoid robot. Besides, the humanoid robot is housing the servos and manufactured with light-weight Aluminum 
brackets which represent the structure of the humanoid [4].

In-Seok Kim et al. proposed a stabilization method for dynamic walking of a humanoid with real-time optimization of capture point (CP) trajectories [5]. Chengju Liu et al. developed a foot positioning compensator FPC for a bipedal robot to retrieve the stability during continuous walking [6]. Ali Fawzi and Ahmed AbdulHussein proposed a three masses linear inverted pendulum model to confirm the robust stability for 17 degrees of freedom (DoF) bipedal robot [7].

In addition to this introduction, this paper contains four other sections. Section 2 presents the theoretical bases of the work. The proposed methodology is fully explained in section 3. The obtained results and their corresponding discussions are included in section 4. Finally, section 5 concludes this paper.

\section{THE THEORETICAL BASES}

In this section, the gait cycle for the bipedal robot and KHR-2HV simulation model are discussed in detail. Also, the forward kinematics model and stability control method of the robot has been presented in this section.

\subsection{Gait cycle of real bipedal robot}

There are two types of bipedal robot walking, the first type is the quasi- dynamics walking and the second type is static walking. The first type requires less energy and has a faster walking speed. For the better walking of the bipedal robot, the bipedal robot must walk in the quasi-dynamic walking [8].

In the case of the second type, the center of mass (CoM) is always between the feet area on the ground when the feet in the double support phase. Walking steps are further divided into six phases as shown in figure (1) [8]:

- $\quad$ Phase one- Double Support Phase (DSP): This phase included both legs on the ground and the projection $(\mathrm{CoM})$ is between the feet on the floor.

- Phase two- Single Support (Pre-Swing): The pre-swing phase happens when the heel of the rear foot is lifting from the floor but the robot is still in (DSP).

- $\quad$ Phase three- Single Support (Swing): In this phase, the foot in swing forward and the other is fully supported with the floor.

- Phase four- Post Swing: The raised leg is placed down with the actuation of ankle joints.
- $\quad$ Phase five and Phase six are a projection of phases (two and three). After phase six, the walking continues with a transition to phase one, and the motion continues.

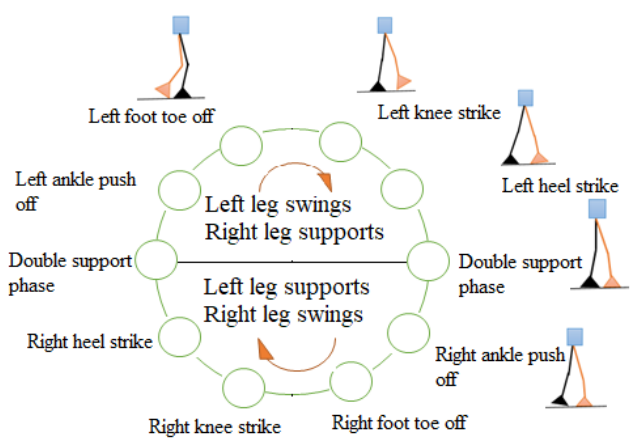

Fig. 1 Gait cycle of bipedal robot [8].

\subsection{Gait cycle of KHR-2HV simulation model}

This section summarizes a brief overview of the software used for training and simulating the gait cycle used in this work. This gives better results when the KHR-2HV simulation model is used for simulating because it is the same design as the bipedal robot used in this work. Webots is a development environment utilized to program, model, and simulates bipedal robots. With Webots, the user can design complex robotics setups, with different robots or several similar in a shared environment.

To measure the (ZMP) for the KHR-2HV simulation model, four load cells were equipped on the sole of each foot [9]. The following footstep commands were characterized for the simulation of a bipedal robot, including a sudden adjustment of foot placement during the (SSP). The gait cycle of the KHR-2HV simulation model is shown in figure (2). To study the gait cycle of the bipedal robot, the walking state or joints movement must be determined to verify the stability:

Walking state $=[$ Right or left roll hip joint, Left or right roll hip joint, Right or left shoulder and arm, Left or right knee, Left or right pitch hip joint, Left or right pitch ankle joint, Right or left roll ankle joint].

For example, to move the left leg in the swing phase:

- In the first stage, the right roll hip joint is moved to the right direction and the left roll hip joint is moved to the left direction, at this moment the right shoulder moved to the backward.

- The second stage, the left knee, left pitch hip joint and left ankle are moved to the backward (This movement gives the left foot push-off). 
- In the third stage, the right roll hip joint is moved to the left direction and the left roll hip joint is moved in the right direction. At this moment, the right shoulder is moved to the forward direction.

- In the fourth stage, all joints belong to the setpoint, but the right shoulder is moved to the forward to move to the right leg with the same above procedures as shown in figure (3) and figure (4) respectively.

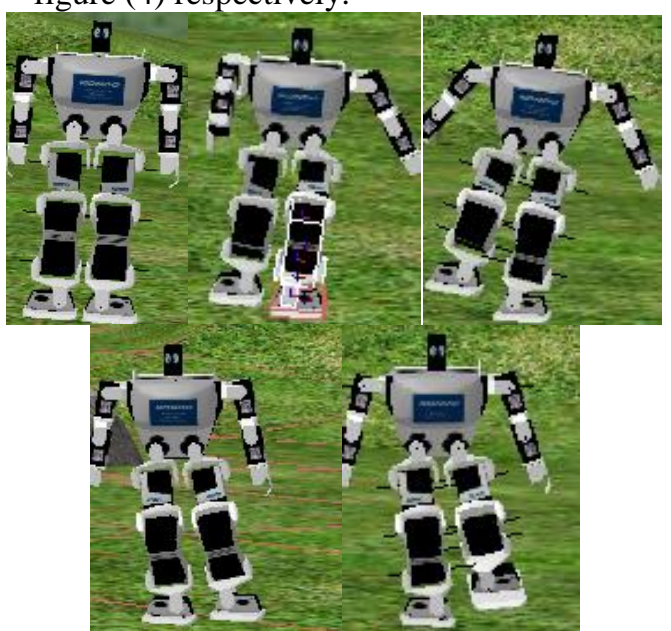

Fig. 2 Gait cycle of KHR-2HV simulation model, ready, right ankle push off, right foot in swing phase, left ankle push off and left foot in swing phase respectively in frontal plane by Webots.

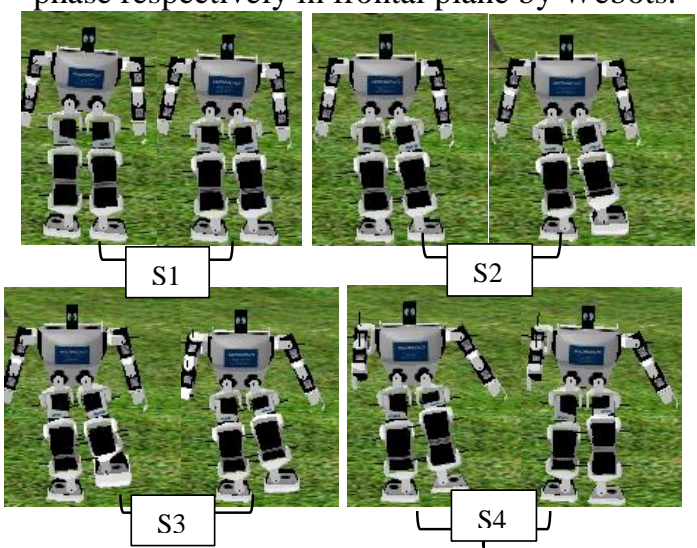

Fig. 3 Snapshots of stages of the KHR-2HV simulation model's left foot in the swing phase in frontal plane by Webots, where $\mathrm{S}$ is the stage number.

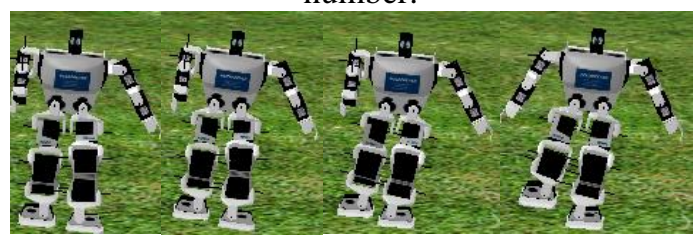

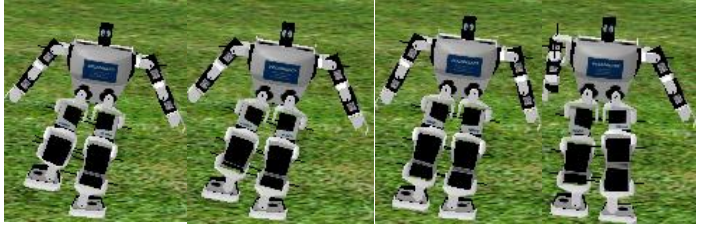

Fig. 4 Snapshots of steps of KHR-2HV simulation model's right foot in frontal plane by Webots.

\subsection{Forward kinematic models for bipedal robot}

In this work, the bipedal robot has seventeen (DoFs) in which five (DoFs) for each leg (one servomotor in each knee joint, two servomotors in each hip joint, and two servomotors in each ankle joint). It is a schematic construction of a bipedal robot as shown in figure (5). The knee joint of both legs has one (DoF) (pitch motion), the hip joint has two (DoFs) (the pitch and roll motion) possible and the ankle joint has two (DoFs) (the pitch and roll motion). Trajectory planning for the swing leg in the (SSP) is achieved by Cartesian coordinates [10]. The joint factors are achieved by the inverse kinematics at each point of time.

A cycloid path in two axes, $\mathrm{z}$-axis (forward) and X-axis (upward) and is estimated for the swing leg trajectory in the (SSP) as shown in figure (6) and discussed below [1]:

$z=c(\emptyset-\sin \emptyset)-\left(\frac{S L}{2}\right)$
$x=c(1-\cos \emptyset)$

Where $\varnothing$ is the rotation angle,

$S L\left(D_{s}\right)$ is step length and

Constant, $c=D_{s} / 2 \pi$.

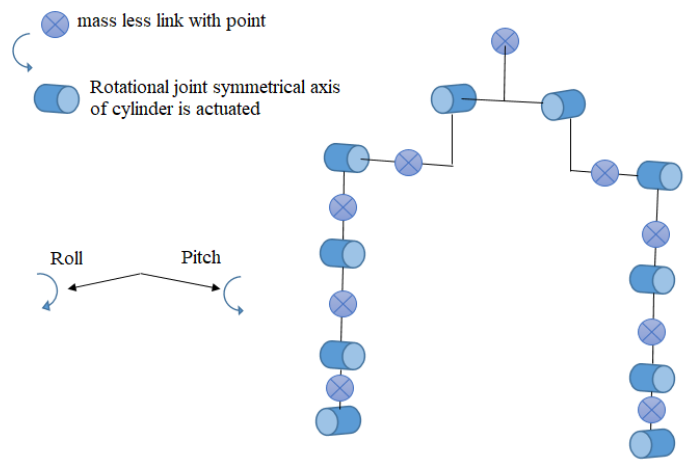

Fig. 5 Schematic overview of anatomical kinematic structure of bipedal robot [10].

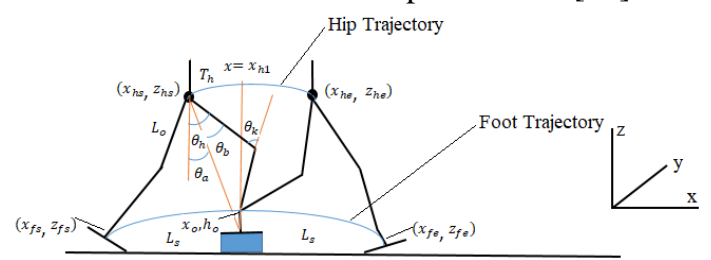

Fig. 6 Swing leg trajectory for walking. 
If both hip trajectory and foot trajectories are identified, all link trajectories of the humanoid will be found by the dynamical model or kinematic configuration. The situation of both legs in the lateral direction is fixed when the humanoid moves in the forward. Joint trajectories are estimated by the inverse kinematics. Motion planning includes the motion of the feet and body. The walking of a bipedal walking robot can be estimated by controlling the foot and hip trajectories.

The stability can be investigated by determining the zero moment point criteria. A cubic polynomial is used to control the sagittal motion [1]. A walking cycle can be divided into two phases, (SSP) and (DSP). In the (SSP), one leg supports the weight of the humanoid while the other leg is moving on the air from forward to backward, at the same time the hip moves along a trajectory $T_{h}$ as illustrated in figure (6). Figure (7) illustrates some important parameters of walking trajectory for a bipedal robot and the walking cycle divided into:

1- (DSP): In this phase, the humanoid is balanced where both feet are in contact with the floor.

2- (SSP): It defines when one leg moves from rear to front and the other is in contact with the ground [1].

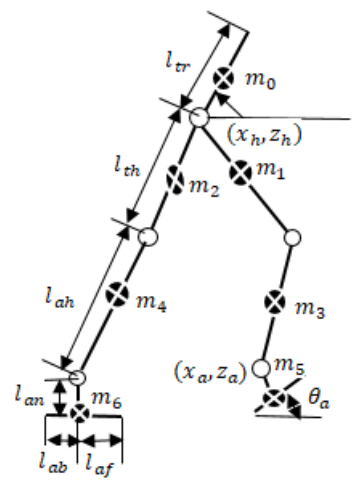

Fig. 7 Model of the bipedal robot [1].

\subsubsection{Trajectory planning of foot joint}

Regarding that the period necessary for one walking step is $T_{c}$, the time of $k$-th step walking is from $\mathrm{KT}_{c}$ to $(\mathrm{K}+1) T_{c}, \mathrm{~K}=1,2, \ldots$ [1]. For simplifying the calculations, the $k$-th step of walking starts with the left foot' heel leaving the floor at $t=\mathrm{KT}_{c}$, and the ends with the left foot's heel come into contact with the floor at $t=(\mathrm{K}+$ 1) $T_{c}$ are defined. The right foot trajectory is the same as the left foot trajectory except for a $T_{c}$ delay.

At the start of the double support phase, especially for high speed walking when the entire sole of the swing foot suddenly touching the floor, the $(\mathrm{CoM})$ will move into the central part of the swing foot in a very short time. When the impact force between the ground may become considerable at the pre-swing phase, the bipedal robot easily tends to fall when the sole of the swing foot. Also, at the starting of the (DSP), the foot slope in the pre-swing phase $\theta_{a}(\mathrm{t})$ is not zero and the heel can put down first before the whole sole arrives the floor.

Also, at the (DSP), the impact force can become insignificant and the (CoM) can move smoothly from the heel to the toe [1]. The rear foot should leave the floor with an appropriate foot slope particularly for high-speed walking to move the body forward. Let $q_{f}$ and $q_{b}$ be the preferred swing foot slope angles as it lands and leaves on the floor as shown in figure (8). The following constraints are [1]:

$\theta_{a}\left(\mathrm{~K} T_{c}+T_{d}\right)=q_{b}$

$\theta_{a}\left(\mathrm{~K} T_{c}+T_{c}\right)=q_{f}$

Where $T_{d}$ represents (DSP) period [1]

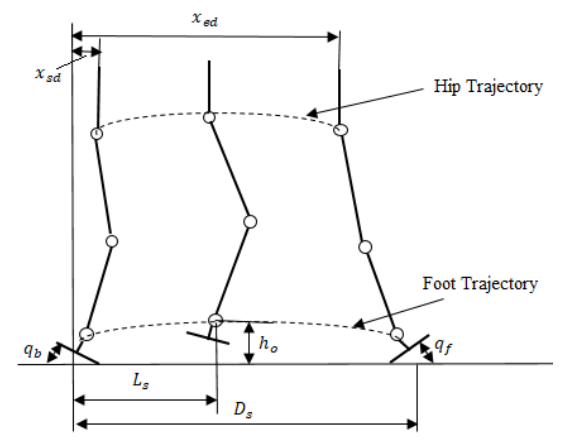

Fig. 8 Parameters of walking pattern [1].

Take into consideration the estimation of minimum energy, where it's important to raise the swing phase to at least a confirmed height in the case of environments or rugged terrain in the existence of obstacles. On the other hand, it is required that the humanoid robot can move without rising its swing phase. Let $\left(L_{s}, h_{o}\right)$ be the values of the highest point of the trajectory of the swing leg as shown in figure (8), the following limitation must be fulfilled [1]:

$X_{a}\left(\mathrm{~K} T_{c}+t_{o}\right)=\mathrm{K} D_{s}+L_{s}$

$Z_{a}\left(\mathrm{KT}_{c}+t_{o}\right)=h_{o}$

$D_{s}$ indicates the length of one step when the swing leg is at its highest point, and $\mathrm{KT}_{c}+t_{o}$ indicates the time as shown in figure (8). From assuming that the whole sole of the left leg arrives the floor, Eq. (3), and Eq. (4) at $t=\mathrm{KT}_{c}$ and $t=$ $\left((\mathrm{K}+1) T_{c}+T_{d}\right)$, the following constraints are [1]:

$\theta_{a}(t)=\left\{\begin{array}{lr}q_{s} & t=\mathrm{K} T_{c} \\ q_{b} & t=\mathrm{KT}_{c}+T_{d} \\ q_{f} & t=\mathrm{K} T_{c}+T_{d} \\ q_{e} & t=(\mathrm{K}+1) T_{c}+T_{d}\end{array}\right.$ 
The slopes of the floor surface are $q_{s}$ and $q_{e}$ at the contact points (in particular $q_{s}=q_{e}=0$ in the case of the level floor. From kinematic constraints and Eq. (3), Eq. (4) and Eq. (5), the following constraints must be satisfied [1]:

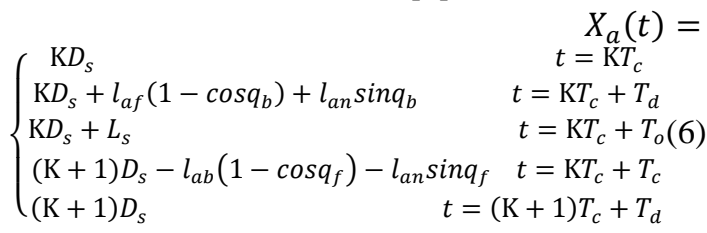$$
\begin{aligned}
& Z_{a}(t)= \\
& \left\{\begin{array}{lr}
l_{a n} & t=\mathrm{K} T_{c} \\
l_{a n} \cos q_{b}+l_{a f} \operatorname{sinq}_{b} & t=\mathrm{K} T_{c}+T_{d} \\
h_{o} & t=\mathrm{K} T_{c}+T_{o} \\
l_{a n} \cos q_{f}-l_{a b} \sin q_{f} & t=\mathrm{K} T_{c}+T_{c} \\
l_{a n} & t=(\mathrm{K}+1) T_{c}+T_{d}
\end{array}\right.
\end{aligned}
$$

\subsection{Stability control method}

Zero moment point (ZMP) is a method used to control the stability of a bipedal robot. Also, the law of the (ZMP) displacement may be performed as follows: at the beginning of phase I the (ZMP) below the heel; at the end of phase I, it jumps to the foot center; when the phase II is finished, it's changed below the toes; at the end of the half-phase the (ZMP) jumps below the other leg which is now being in support phase on the floor. Three various laws and five various laws of the (ZMP) displacement in the (DSP) and (SSP) respectively, corresponding to a half-step (T). To estimate (ZMP) for a bipedal robot, forward kinematics hypotheses that have to be made [11]:

a) The bipedal walking robot consists of $n$ links.

b) All kinematic assumptions, such as link orientation, the position of $(\mathrm{CoM})$, and velocities are identified and estimated by the forward kinematics.

c) The ground is motionless and rigid.

d) The foot can't slide over the ground.

e) The joint is actively actuated.

Under these restrictions, the first thing to estimate is the total mass $m_{\text {total }}$ of the bipedal robot and $p$ is the distance from the center of the link to the baseframe-origin:

$\mathrm{m}_{\text {tot }}=\sum_{i=1}^{n} \mathrm{~m}_{i}$

$p_{\text {CoM }}$ is the distance from equivalent (CoM) to the base-frame-origin as shown in figure (9).

$\mathrm{P}=\sum_{i=1}^{n} m_{i} \dot{p}_{i}$

$\mathrm{H}=\sum_{i=1}^{n}\left\{\mathrm{p}_{i} \times \mathrm{m}_{i} \dot{\mathrm{p}}+I_{i} \omega_{i}\right\}$

Where $\mathrm{H}$ and $\mathrm{P}$ are the total angular and linear momentum respectively with respect to the origin OXYZ.
Where $\omega_{i}$ and $I_{i}$ are the angular velocity and the inertia tensor of the $i$-th link respectively with respect to origin $\mathrm{O}_{\mathrm{XYZ}}$.

For $I_{i}$ the following equation holds:

$I_{i}=R_{i} I_{i} R_{i}^{T}$

Where $R_{i}$ is the rotation matrix of $\mathrm{i}$-th link with respect to the origin $\mathrm{O}_{\mathrm{XYZ}}$ connected to their links. $\dot{\mathrm{H}}$ and $\dot{\mathrm{P}}$ are the time derivative of angular momentum and linear momentum (being a moment and a force), respectively. They can be stated as:

$\dot{\mathrm{P}}=\sum_{i=1}^{n} m_{i} \ddot{p}_{i}$

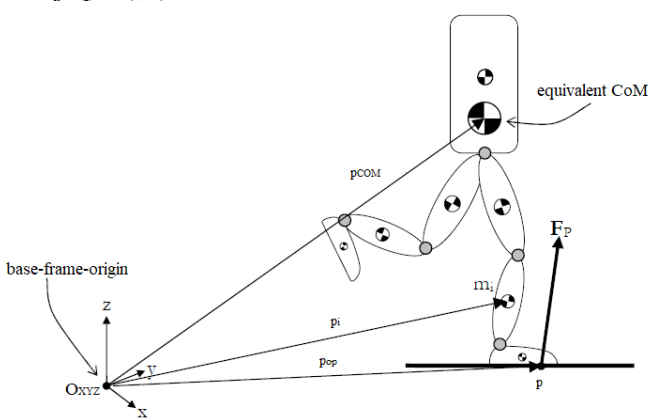

Fig. 9: Schematic 3-D biped model and zero moment point $p$ [11].

$\dot{\mathrm{H}}=\sum_{i=1}^{n}\left(\dot{p}_{\imath} \times\left(m_{i} \dot{p}_{i}\right)+p_{i} \times\left(m_{i} \ddot{p}_{i}\right)+I_{i} \dot{\omega}_{i}+\right.$ $\left.\omega_{i} \times\left(I_{i} \omega_{i}\right)\right)$

Where $\dot{p}_{i} \times\left(m_{i} \dot{p}_{i}\right)=0$ because $\dot{p}_{i}$ and $\left(m_{i} \dot{p}_{i}\right)$ are parallel (note that $\left(m_{i} \dot{p}_{i}\right)$ a scalar multiplication of $\left(\dot{p}_{i}\right)$ [11]. With these assumptions the following holds:

$F_{p}=-F_{A}=\dot{\mathrm{P}}-m_{\text {tot }} g$

$M_{o}=\dot{\mathrm{H}}-p \times m_{\text {tot }} g$

Where, as said earlier $M_{o}$ and $F_{p}$ are the moment and external forces that characterize how the ground is reacting to the biped w.r.t. base-frameorigin. $F_{A}$ is the force that the biped is acting upon the floor and $g$ is the gravitational acceleration. Also, the $M_{o}$ is:

$M_{o}=p_{o p} \times F_{p}+M_{p}$

Where $p_{o p}$ is the vector from the base-frame-origin to point $p$ and $M_{p}$ is the moment at $p$. Because $M_{p}$ is on the point $p$, being either (ZMP), its $M_{p}=\left[\begin{array}{ll}0 & 0\end{array}\right.$ $M_{z}$ ] [11]. Now, Eq. (14) is substituted into Eq. (15) resulting in:

$M_{p}=\dot{\mathrm{H}}-p_{C o M} \times m_{\text {tot }} g+\left(\dot{\mathrm{P}}-m_{\text {tot }} g\right) \times p_{o p}$

(16)

From this, the distance from location of the (ZMP) to the origin $\mathrm{O}_{\mathrm{XYZ}} \quad p_{z m p}=p_{o p}=$ $\left[x_{Z M P}, y_{Z M P}, z_{Z M P}\right]$ can be calculated:

$x_{Z M P}=\frac{m_{\text {tot }} g_{z} p_{\operatorname{CoM} x}+z_{Z M P} \dot{\mathrm{P}}_{x}-\dot{\mathrm{H}}_{y}}{m_{\text {tot }} g_{z}+\dot{\mathrm{P}}_{z}}$

$y_{Z M P}=\frac{m_{t o t} g_{z} p_{C o M y}+z_{Z M P} \dot{\mathrm{P}}_{y}-\dot{\mathrm{H}}_{x}}{m_{\text {tot }} g_{z}+\dot{\mathrm{P}}_{z}}$

Where $x_{Z M P}$ and $y_{Z M P}$ are the distances from ZMP to the base-frame-origin about $\mathrm{x}$-axis and $\mathrm{y}$-axis 
respectively. Remind that $z_{Z M P}$ is the height of the ground. When the $\mathrm{x}$-axis and $\mathrm{y}$-axis are situated on the ground $z_{Z M P}$ becomes zero [11].

Huang et al. (2001) [11] hypothesized that $z_{Z M P}=$ 0 , and stated the following equation for deriving the position of the ZMP:

$$
x_{Z M P}=\frac{\sum_{i=1}^{n}\left(m_{i}\left(p_{i x}\left(\ddot{p}_{i z}+g_{z}\right)-p_{i z}\left(\ddot{p}_{i x}+g_{x}\right)\right)-I_{i y} \omega_{i y}\right)}{\sum_{i=1}^{n} m_{i}\left(\ddot{p}_{i z}+g_{z}\right)}
$$

$$
y_{Z M P}=\frac{\sum_{i=1}^{n}\left(m_{i}\left(p_{i y}\left(\ddot{p}_{i z}+g_{z}\right)-p_{i z}\left(\ddot{p}_{i y}+g_{y}\right)\right)-I_{i x} \omega_{i x}\right)}{\sum_{i=1}^{n} m_{i}\left(\ddot{p}_{i z}+g_{z}\right)}
$$

The foot rotation must keep with the support polygon to guarantee no foot rotation, regardless of the ground projection center of mass (GCoM). The foot rotation indicator (FRI) $f$ is minimized the ground projection center of mass location and a dynamics-based method for a stationary legged robot that has two legs. The minimum distance is an estimation of the instability of the bipedal robot when the foot rotation indicator point is out the footprint. The estimation of the instability of the bipedal robot of the support limit from the present situation of the foot rotation indicator point can be identified by within the foot point and by the balance boundary of a humanoid against foot rotation as shown in figure (10) [12]. The robot is stable when the (FRI) $f$ is situated within support polygon and the robot is unstable when the (FRI) outside the support polygon as shown in figure (10-a) and (10-b) respectively.
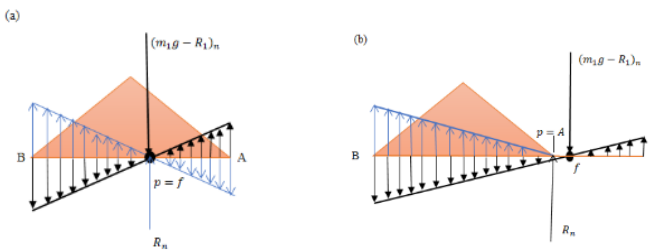

Fig. 10: The value of the derivative angular momentum a point on the support polygon region [12].

\section{THE PROPOSED METHODOLOGY}

This section explains the proposed research, including conceptual design for the real two types of (DoF) bipedal robots, 10 (DoFs) and 17(DoFs) bipedal robots are utilized in this work. A humanoid robot can be commonly explained as the kinds of an autonomous system this can simulate human walking motion with maintaining postural stability through the motion. The design of a bipedal robot is very necessary for the available performance of the bipedal robot, especially the weight of the system imposes physical limits.
The humanoid structure is highly appropriate for use in the human environment due to its advantages in the ability to be applied as human substitutes and obstacle avoidance [13]. A bipedal robot has five (DoFs) for each leg, where two (DoFs) at the ankle joint, two (DoFs) at the hip joint, and one (DoF) at the knee joint as shown in figure (11). The bipedal robot can be classified into three blocks servo controller board, control unit, and the servomotors worked as actuators. Figure (12) illustrates 10 degrees of freedom (DoFs) bipedal robot.

The control unit transmits the control code to the servomotor board depend on the movement needed. The servomotor controller produces pulse width modulation (PWM) signal with period time pulse width depending on the instruction sustained thereby rotating the servos with angles and speed as needed. The following experimental setup will show the components present in a humanoid robot. The robot components is divided into two parts, hardware and software. Each section will illustrate in detailed a component that used for making a bipedal robot.

Arduino microcontroller is typically computer type electrical circuitry; however, a bipedal walking robot can also be programmed by a connected computer system. It is a computer type circuitry commonly placed on one chip or (PIC) Programmable Integrated Circuit, which has a memory, optional math processing, (CPU) Central Processing Unit and bus. Both sensors and motor are present in the robot, which are controlled by the microcontroller.

The technical specifications of the Arduino microcontroller are (operating voltage: 5volts, Analog input pins: 6, flash memory: $32 \mathrm{~KB}$, clock speed:16 MHz, size: $68.6 \mathrm{~mm} * 53.4 \mathrm{~mm}$ and weight $25 \mathrm{~g}$ ). The high torque servomotor has three wires that classified according to the colors (orange (PWM), red (VCC), and brown (ground)). Where PWM wire is connected to (I/O) pins, VCC is connected with a 5Volt pin in power supply, and Ground is connected with the (GND) pin in the power supply. The (PWM) method enables a capable electromotor to regulate the direction of rotation and the angular velocity depends on the speed and width of pulses received.

PWM-16 channel servo driver is a sixteen channels - twelve bit Pulse Width Modulation/servomotor driver that will drive up to sixteen servomotors over $12 \mathrm{C}$ with only two pins. Two positions of control input (SCL, SDA, OE) pins and power pins (GND, VCC, $\mathrm{V}^{+}$) on both sides are situated [14]. Both sides of the pins are identical. Sixteen output ports are there in the PCA 
96685-servo driver. Each port has three pins: (GND), VCC, and the pulse width modulation pin. To connect the PCA 96685 with a microcontroller as shown in figure (13), the steps of the connection are: 5 Volts with VCC, Analog 5 with (SCL), (GND with GND) and (Analog 4 with SDA) [14]. Be sure to attach the signal wire (commonly white or yellow) with the top row, the VCC wire (usually red) with the middle row, and the ground wire (commonly brown or black) with the bottom row in the (PWM) 16 driver was drawing by the Fritzing program as shown in figure (13).

LOBOT LSC-32 model is a device that is used to programming the servomotors by Lobot Servo Control Program as shown in the figure (14). The servomotor interface of this type has overcurrent protection. To avoid a short circuit during the running, first, connect the servomotors to the LSC-32 servo controller and then connect the battery to the servo controller. This model was chosen because it is one of the most practical ways to describe the gait cycle for the humanoid robot as shown in figure (15). The voltage of the LOBOT LSC-32 model must be delivered between $(5 \mathrm{~V}$ to $9 \mathrm{~V})$. Besides, this type consists of a 32-servo connection port with over current protection.

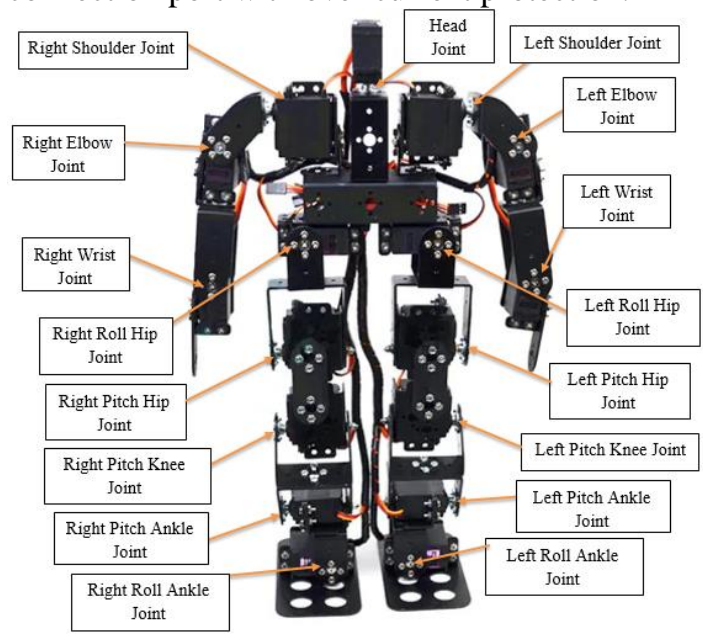

Fig. 11 17DoF bipedal robot.

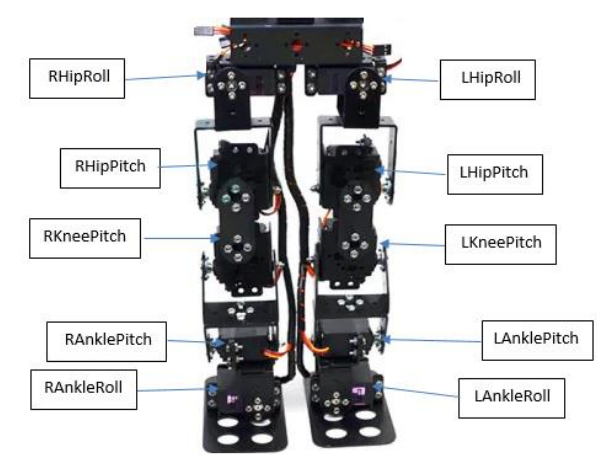

Fig. 1210 DoFs bipedal robot, where $\mathrm{R}$ is right and $\mathrm{L}$ is the left direction.

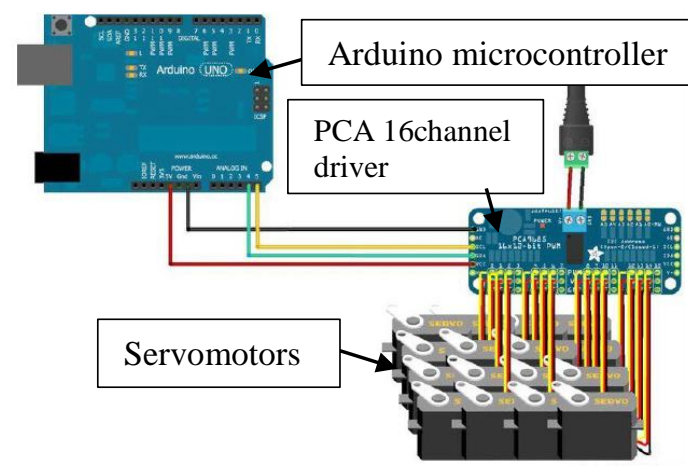

Fig. 13 The connection PCA 16channel driver with Ardino microcontroller [14].

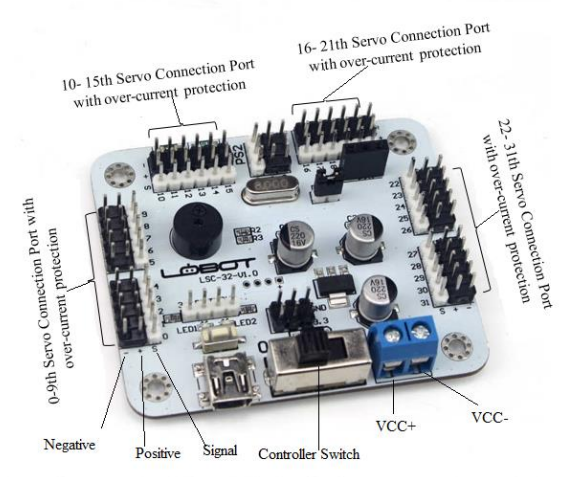

Fig. 14 LOBOT LSC-32 model.

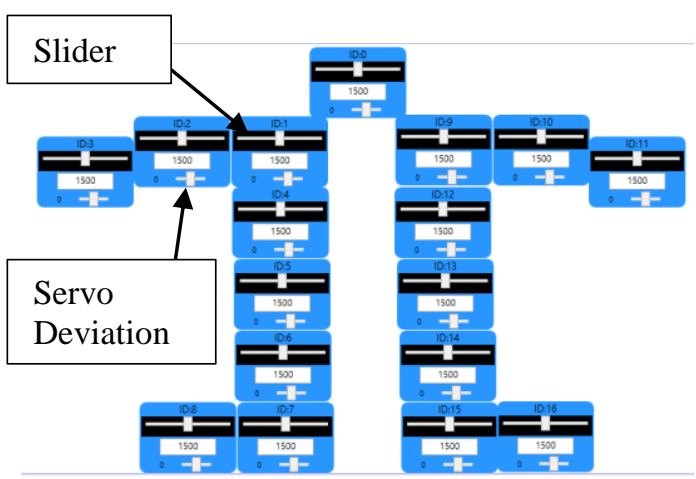

Fig. 15 Bipedal robot by lobot servo control in the DSP.

\section{RESULTS AND DISCUSSIONS}

In this section, simulation and experimental results are discussed. The simulation results are an indicator of the experimental results. Simulations were used to indicate the experimental results. After the installation of Lobot Servo Control software with the servo controller is connected to the PC, the interface indicator turn green denotes that connecting is successful. Now, the humanoid robot is built by servo window action as shown in figure (15).

The servomotor slider can be drag freely between 500 to 2500 , where it can visually show the rotation position of the servomotor at this time. 
Besides, there is a deviation of the servo below the slider and it begins from (-100 to 100$)$. To simulate the experimental results with the simulations, the calibration curve is used as shown in Eq. (20). The equation of the calibration is

Angle $\left(^{\circ}\right)=0.18 \times$ Slider Value

A bipedal robot could start falling before (ZMP) reaches the convex hull of the support area and approaching zero moment point to the convex hull could occur when the falling of a robot is unavoidable. The bipedal model used in this work consists of two legs and a trunk connecting them, where two joint axes are positioned at the hip, one joint at the knee and two joints are at the ankle.

The movement of the leg and arm are utilized as the important parts of the kinematics. Figure (16) shows the differences in the pitch direction of servomotors because of the differences in the connection way of servomotors to the joints. Kinematic obsession is the trajectory-based method and becomes of its extensive utilize in bipedal robots. The important common method to the bipedal walking robot has been to realize the human arm and leg position over time.

A gait cycle divided into two parts and encompasses a number of the steps as shown in figure (1). The gait cycle for this humanoid was proposed by using an LSC-32driver and approaching statically stable gain. First, this work is initially carried out by trial and error. As mentioned, there are two types of walking stability, dynamic and static walking. During the walking, the speed in the experiments of transition and/or change in balance assumes that the humanoid is statically stable at all moments and extremities from one stable to the other stable region would result in the gait. To control the stability of the bipedal robot at any time in the walking, zero moment point must remain in a balanced area.

A gait cycle, which is described above is split into five steps. Besides, gait cycles are realized when the steps are repeated. The design of the ankle joints, allows for turning and halting, rather than exhibits the system more time-efficient and makes a closer resemblance to the human walks, which permits the bipedal robot to turn while walking. When the bipedal robot is turning in any direction, the roll and pitch ankle joints play the major rule. Instead of an ineffectual $90^{\circ}$ turn, the bipedal robots can make an obtuse and acute turn based on their requirements.

When tested, bipedal robot motion can be divided into a series of stable states and near the stable. From the first state to the other state, the speed of transition and/or change in the stability extremities would result in the gait being dynamic or static. The bipedal robots are statically stable in the static walking during the walking and hypothesize at all moments. Walking gaits encompass several steps as shown in figure (17). The first stage comprises the initial state begins when the humanoid balanced and is ready to walk. The change in (CoM) to its right or left is labeled as a second stage. The third stage completes the process of the system it is first. The fourth stage comprises the changing its (CoM) back to the center of the humanoid [14].

First, the kinematic of the bipedal robot is studied for ten (DoFs) bipedal as shown in figure (5). First, when the bipedal robot is in (DSP), the (ZMP) must be between the feet to confirm the stability and equivalent with (CoM) [15]. At the beginning of phase I the (ZMP) below the heel; at the end of phase I, it jumps to the foot center; at the end of phase II it's changed below the toes; at the end of the half-phase, the (ZMP) jumps below the other foot which is now being in contact with the ground [16].

For control of the stability of the bipedal robot, (ZMP) must be estimated theoretically and experimentally. Four load cells are mounted on the sole of each foot to measure (ZMP) while walking. The weight and height of the bipedal robot are $1.8 \mathrm{~kg}$ and $38 \mathrm{~cm}$ respectively. A bipedal robot has seventeen degrees of freedom (one (DoF) for the head, ten (DoFs) for the legs and six (DoFs) for the arms). Table (1) shows the magnitudes of the angles of the right leg, and left leg in the swing phase for ten (DoFs) bipedal robots.

Table (2) gives some important simulation parameters for the step of the bipedal robot. Experimental results are carried out with this robot model, coordination and control mechanism and references generated are depend on simulation results. The dimensions of the leg are divided into three parts, the upper part has a roll hip joint, the second part has pitch hip joint and knee joint and the lower part has the ankle joints. To study the foot trajectory of the bipedal robot, the forward kinematic model must be used. The height of the foot is $1.2 \mathrm{~cm}$, therefore the foot trajectory begins from $1.2 \mathrm{~cm}$ as shown in figure (18).

First, when the robot is programmed to walk, the robot is falling before completing the first step because the foot rotation indicator (FRI) exists it and the rotation of support foot happens as (ZMP) reaches the boundaries of the support area. To simplify the analysis, the $k$-th walking step begins when the right foot at toe off phase or the heel of the right leg leaving the floor at $t=0.18 \mathrm{~s}$, and the ends when the right foot at strike phase or the heel of the right leg arriving the ground at $t=$ $1.25 \mathrm{~s}$ to complete one step. The left foot trajectory 
is the same as the right foot trajectory except for the step period $T_{c}$.

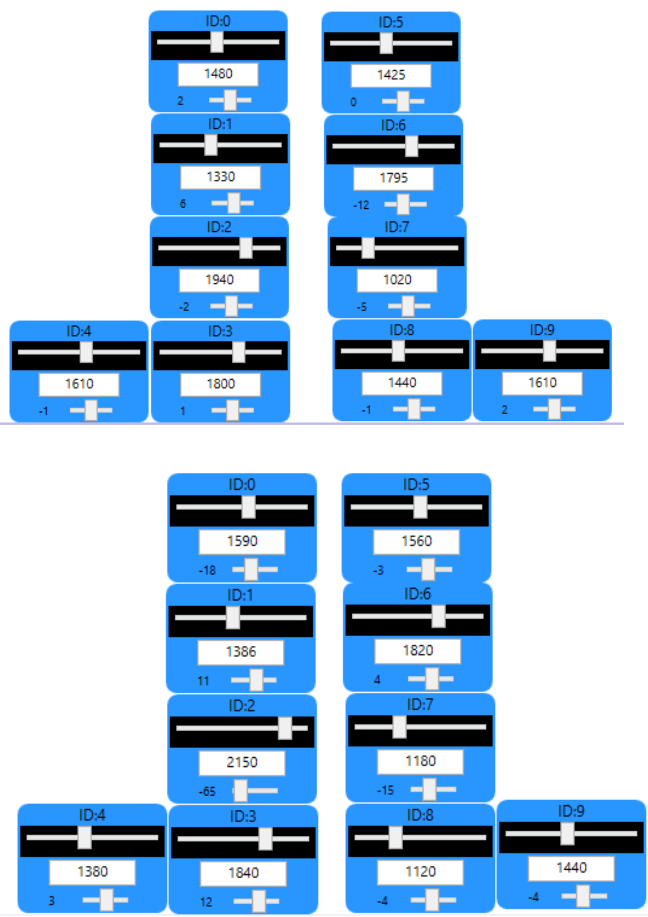

Fig. 16 Left leg and right leg slider values in swing phase for 10 DoFs bipedal robot.

The bipedal robot easily tends to fall when the impact force between the sole of the swing leg and the ground may become very large in the pre-swing phase. At the starting of the double support phase, the (CoM) will move to the central part of the swing foot for three seconds when the whole sole of the swing leg will suddenly come into touch with the floor to complete one cycle. On the other hand, the heel can put down first before the whole sole arrives at the floor when the foot slope in the pre-swing phase $\theta_{a}(\mathrm{t})$ is not zero at the starting of the (DSP).

The cubic polynomial foot trajectory equation is $y=4 \times 10^{-16} z^{3}-0.0433 z^{2}+$ $0.4329 z+0.7619$ with regression 0.9276 for 10 degrees of freedom bipedal robot as shown in figure (18). From this equation, the bipedal robot walks with the smaller $h_{o}$ because it is moved on the floor. With hips trajectory and feet trajectory, inverse kinematic is used to determine the joint angles of the support and swing leg. The information of the bipedal robot system to estimate joints angles through inverse kinematic.

The joints angles of the swing leg and the joints angles of supporting leg are estimated by inverse kinematics. Joint trajectories are estimated by the inverse kinematics. Motion planning includes the motion of the feet and body. The walking of a bipedal walking robot can be estimated by controlling the foot and hip trajectories. The stability can be investigated by determining the zero moment point criteria. Table (3) shows the masses and dimensions of the 17 (DoFs) links.

Table 1: Angles of right leg and left leg in swing phase for 10 (DoFs) bipedal robot.

\begin{tabular}{|c|c|c|}
\hline $\begin{array}{c}\text { Left Leg in } \\
\text { Swing } \\
\text { Phase in }\left(^{\circ}\right)\end{array}$ & $\begin{array}{c}\text { Right Leg in } \\
\text { Swing Phase } \\
\text { in }\left(^{\circ}\right)\end{array}$ \\
\hline $\begin{array}{c}\text { Jeft Roll Hip } \\
\text { Jitch Hip } \\
\text { Joint }\end{array}$ & -13.5 & 10.8 \\
\hline $\begin{array}{c}\text { Left Knee } \\
\text { Joint }\end{array}$ & -86.4 & 57.6 \\
\hline $\begin{array}{c}\text { Left Pitch } \\
\text { Ankle Joint }\end{array}$ & -10.8 & -57.6 \\
\hline $\begin{array}{c}\text { Left Roll } \\
\text { Ankle Joint }\end{array}$ & 19.8 & -10.8 \\
\hline $\begin{array}{c}\text { Right Roll } \\
\text { Hip Joint }\end{array}$ & -3.6 & 16.2 \\
\hline $\begin{array}{c}\text { Right Pitch } \\
\text { Hip Joint }\end{array}$ & -30.6 & -20.52 \\
\hline $\begin{array}{c}\text { Right Knee } \\
\text { Joint }\end{array}$ & 79.2 & 117 \\
\hline $\begin{array}{c}\text { Right Pitch } \\
\text { Ankle Joint }\end{array}$ & 54 & 61.2 \\
\hline $\begin{array}{c}\text { Right Roll } \\
\text { Ankle Joint }\end{array}$ & 19.8 & -21.6 \\
\hline
\end{tabular}
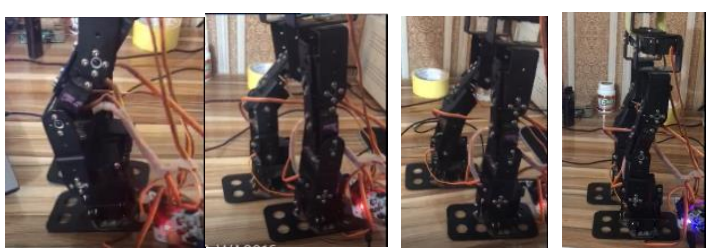

Fig. 17 Gait cycle of bipedal robot for $10 \mathrm{DoFs}$ in sagittal plane.

A cubic polynomial is used to control the sagittal motion. A walking cycle can be divided into (SSP) and (DSP) as shown in figure (1). The kinematic model of the bipedal robot is described as Sec. (2.3). The $\mathrm{x}$-axis points forward, the $\mathrm{z}$-axis points upward and the y-axis is the cross product of the $\mathrm{x}$-axis and z-axis. The cubic spline confirmed the smooth walking trajectory and smooth zero moment point.

Table 2: Important simulation parameters in step for 10 DoFs robot.

\begin{tabular}{|l|l|}
\hline Parameter & Value \\
\hline Step height & $0.8 \mathrm{~cm}$ \\
\hline Step period & $1.25 \mathrm{~s}$ \\
\hline
\end{tabular}




\begin{tabular}{|c|c|}
\hline $\begin{array}{l}\text { Time interval of the DSP } \\
T_{d}\end{array}$ & $1.75 \mathrm{~s}$ \\
\hline $\begin{array}{l}\text { Length from the ankle to } \\
\text { the toe } l_{a f}\end{array}$ & $7.2 \mathrm{~cm}$ \\
\hline The height of the foot $l_{a n}$ & $1.2 \mathrm{~cm}$ \\
\hline $\begin{array}{l}\text { The length from ankle joint } \\
\text { to the heel } l_{a b}\end{array}$ & $5 \mathrm{~cm}$ \\
\hline $\begin{array}{l}\text { Step length }\left(D_{s}\right) \text { in the } \mathrm{Z} \text { - } \\
\text { direction }\end{array}$ & $6 \mathrm{~cm}$ \\
\hline $\begin{array}{l}\text { Distance between feet in the } \\
\text { X-direction }\end{array}$ & $3 \mathrm{~cm}$ \\
\hline $\begin{array}{l}\text { The distance from CoM to } \\
\text { the ground }\end{array}$ & $28 \mathrm{~cm}$ \\
\hline
\end{tabular}

Before the bipedal robot completes the first step, the robot is falling because the foot rotation indicator (FRI) point $f$ does not equivalent to the center of pressure (CoP) [12]. Currently, two various approaches are signifying followed in work to realize bipedal locomotion robot: a walking pattern concerning the perception of several numbers of trajectories is either estimated online, the humanoid chooses one of these per calculated trajectories concerning the position, and the actual intention [17].

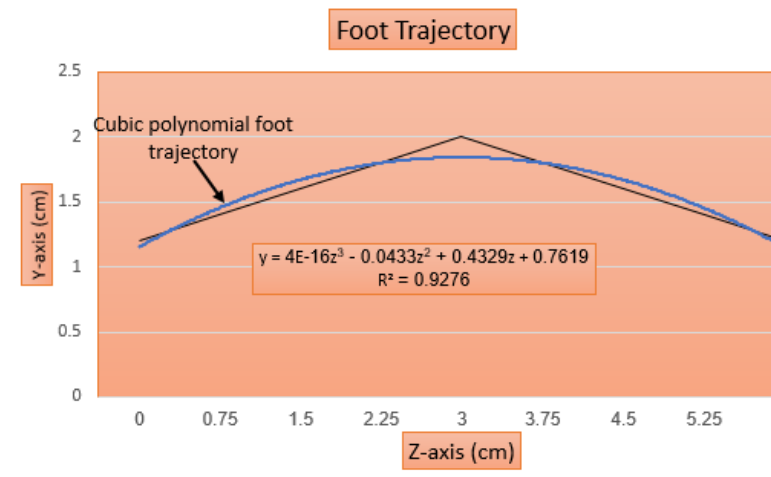

Fig. 18 Cubic polynomial foot trajectory of 10 DoFs bipedal robot.

The gait cycle needs to confirm some "aesthetic" method, as an appropriate the movement, such as energy efficiency, effectiveness, and smoothness, online generation of gait cannot be estimated by computing distinct formulations and requires recursive optimization of the trajectory and trajectory for a humanoid is subject to many constraints. Table (3) shows the masses and dimensions of the 17 (DoFs) bipedal robot.

Table 3: Masses and dimensions of the 17 DoFs bipedal robot links.

\begin{tabular}{ll|c} 
Link & $\begin{array}{l}\text { Dimensions } \\
(\mathbf{L} * \mathbf{W} \mathbf{H})\end{array}$ & Mass (g) \\
$\mathbf{c m}$ & in & \\
\hline Arm & $6 \times 2.5 \times 20$ & 223 \\
\hline
\end{tabular}

\begin{tabular}{|l|l|l|l|}
\hline Leg & $\begin{array}{l}\text { Upper } \\
\text { part } \\
\text { Middle } \\
\text { part }\end{array}$ & $5 \times 4 \times 5$ & 600 \\
\hline $\begin{array}{l}\text { Lower } \\
\text { part }\end{array}$ & $5 \times 6 \times 7$ & \\
\hline Shoulder & $4 \times 3 \times 6$ & 85 \\
\hline Head & $4 \times 3 \times 6$ & 75 \\
\hline
\end{tabular}

Gaits generation is broken into several tasks: the first is to need the inverse kinematics for computing the position of the parts of the humanoid given a confirm link configuration and the second task is to set up the humanoid kinetics to be capable for simulating the gait cycle of the bipedal robot. The left foot trajectory is the same as the right foot trajectory except for the step period $T_{c}$ because the steps are not equaled and vice versa. Since the (CoM) will move to the central part of the swing foot and the whole sole of the swing foot will suddenly be arriving at the ground or come into attaching with the floor at the starting of the (DSP) (pre-swing phase).

In the pre-swing phase, the foot slope in the pre-swing phase $\theta_{a}(\mathrm{t})$ is not zero at the starting of the (DSP) and the heel can put down first before the whole sole arrives at the floor. On the other hand, the impact force between the sole of the swing foot and the ground may become very large, and the bipedal robot easily tends to fall. The cubic polynomial foot trajectory equation for 17 (DoFs) bipedal robot is $y=-0.000074 z^{3}-0.13 z^{2}+$ $0.671 z+1.1326$ with regression 0.939 as shown in figure (19).

From this equation, the bipedal robot walks with the smaller $h_{o}=1 \mathrm{~cm}$ because it is moved on the floor. Where the foot trajectory begins from the height of the foot $(1.2 \mathrm{~cm})$ as a cubic polynomial equation with step period $1.35 \mathrm{~s}$. Table (4) shows some parameters that used to confirm the foot trajectory for 17 (DoFs) bipedal robots.

Figure (20) gives an overview of the motion of the servomotors for a bipedal robot to confirm the step by the right leg. Also, figure (21) describes the directions of the servomotors of the left leg to get the second step to complete the gait cycle of 17 (DoFs) bipedal robot. The differences of directions the servomotors in the figures belong to the connections of the servomotors with the links. In the initialization, the bipedal walking robot is tending to stability or in a stable position.

Table 4: Some of important simulation parameters in Step for 17 (DoFs) Bipedal Robot

\begin{tabular}{|l|l|}
\hline Parameter & Value \\
\hline Step height & $1 \mathrm{~cm}$ \\
\hline
\end{tabular}




\begin{tabular}{|l|l|}
\hline Step period & $1.35 \mathrm{~s}$ \\
\hline $\begin{array}{l}\text { Time interval of the DSP } \\
\boldsymbol{T}_{\boldsymbol{d}}\end{array}$ & $1.9 \mathrm{~s}$ \\
\hline $\begin{array}{l}\text { Length from the ankle to } \\
\text { the toe } \boldsymbol{l}_{\boldsymbol{a}}\end{array}$ & $7.2 \mathrm{~cm}$ \\
\hline The height of the foot $\boldsymbol{l}_{\boldsymbol{a n}}$ & $1.2 \mathrm{~cm}$ \\
\hline $\begin{array}{l}\text { The length from ankle } \\
\text { joint to the heel } \boldsymbol{l}_{\boldsymbol{a}}\end{array}$ & $5 \mathrm{~cm}$ \\
$\begin{array}{l}\text { Step length }\left(\boldsymbol{D}_{\boldsymbol{s}}\right) \text { in the Z- }- \\
\text { direction }\end{array}$ & $5 \mathrm{~cm}$ \\
\hline $\begin{array}{l}\text { Distance between feet in } \\
\text { the X-direction }\end{array}$ & $2.8 \mathrm{~cm}$ \\
\hline $\begin{array}{l}\text { The distance from CoM } \\
\text { to the ground }\end{array}$ & $28 \mathrm{~cm}$ \\
\hline
\end{tabular}

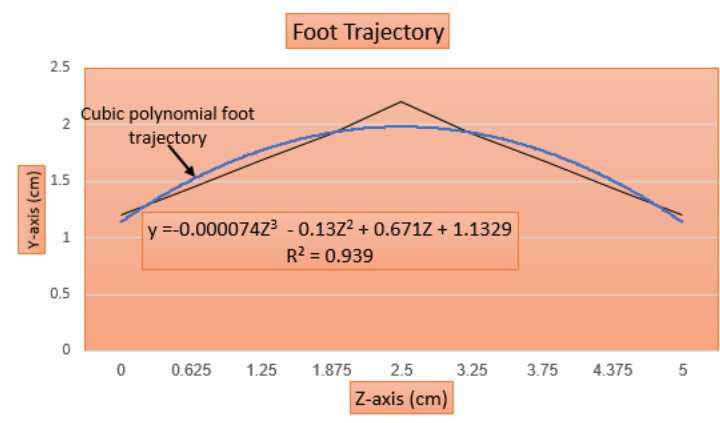

Fig. 19 Cubic polynomial foot trajectory for 17 DoFs bipedal robot.

The initialization is applied to find the mode of walking and the accurate direction. The balance control state involved the changing of the humanoid's $(\mathrm{CoM})$ in a falling position tending towards stability. In the termination, it includes the humanoid belonging to a stability control as it occurs of the movement. Dynamic balance occurred when one foot on the ground and the other foot in the swing phase or the robot's (CoM) is not inside the area between the feet.

Our experiment is, to begin with, a preprogrammed semi-balanced, but parameterized gait for a bipedal robot [17]. Gait factors such as step length, walking speed, the height of leg lift, leaning angle of the torso forward direction, and maximal leaning angle torso sideways as described in table (5). Inverse kinematic was utilized for confirming the joint angles as shown in table (5). With the reference angles for right and left leg in the swing phase as shown in table (5), four steps walking tests were done. Figure (20) and (21) are utilized by depending on the simulation results to confirm the stability and get a faster response of 17 (DoFs) bipedal robots.

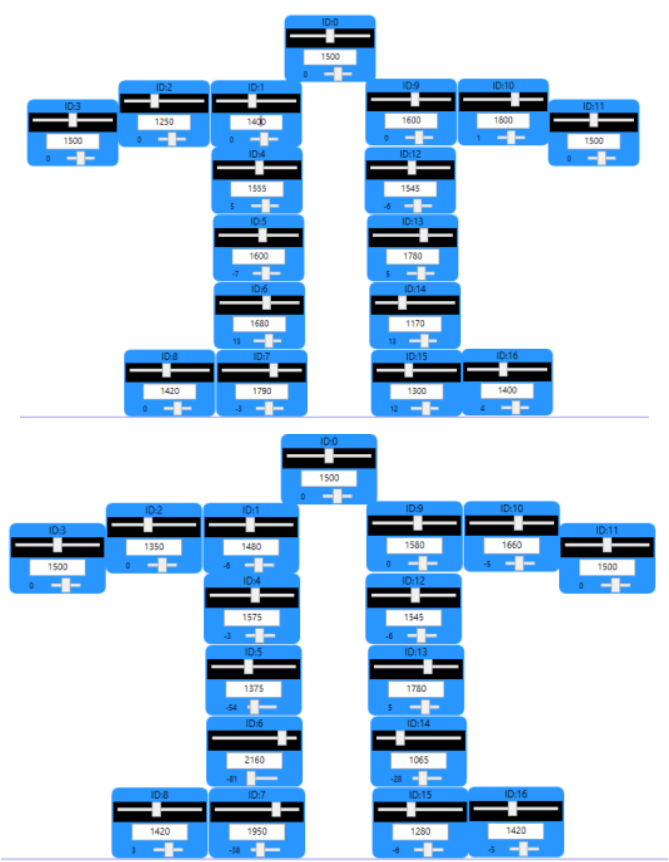

Fig. 20 Experimentally right ankle push off and right leg slider values in swing phase by Lobot

Servo Control for 17 DoFs bipedal robot.

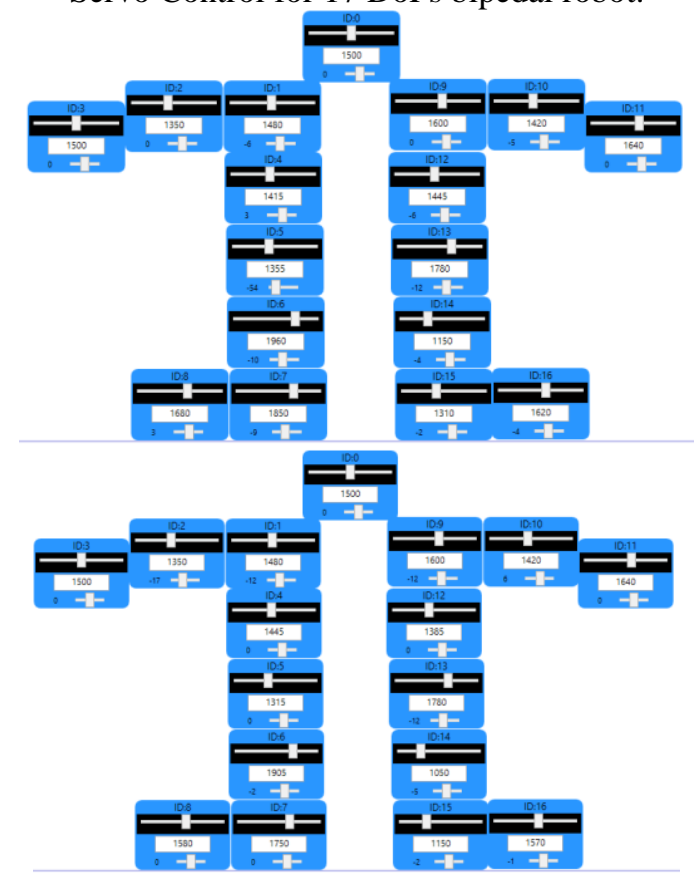

Fig. 21 Experimentally left ankle push off and left leg slider values in swing phase by Lobot Servo

Control for 10 DoFs bipedal robot.

Table 5: Experimentally angles of servomotors for 17 DoFs bipedal robot.

\begin{tabular}{|c|c|c|}
\hline Link & $\begin{array}{c}\text { Left Leg in } \\
\text { Swing } \\
\text { Phase in }\left({ }^{\circ}\right)\end{array}$ & $\begin{array}{c}\text { Right Leg } \\
\text { in Swing } \\
\text { Phase in }\left({ }^{\circ}\right)\end{array}$ \\
\hline Head & 0 & 0 \\
\hline $\begin{array}{c}\text { Left Roll Hip } \\
\text { Joint }\end{array}$ & -20.7 & 8.1 \\
\hline
\end{tabular}




\begin{tabular}{|c|c|c|}
\hline $\begin{array}{c}\text { Left Pitch Hip } \\
\text { Joint }\end{array}$ & 50.4 & 50.4 \\
\hline Left Knee Joint & -81 & -78.3 \\
\hline $\begin{array}{c}\text { Left Pitch Ankle } \\
\text { Joint }\end{array}$ & -63 & -39.6 \\
\hline $\begin{array}{c}\text { Left Roll Ankle } \\
\text { Joint }\end{array}$ & 12.6 & -14.4 \\
\hline $\begin{array}{c}\text { Right Roll Hip } \\
\text { Joint }\end{array}$ & -9.9 & 13.5 \\
\hline $\begin{array}{c}\text { Right Pitch Hip } \\
\text { Joint }\end{array}$ & -33.3 & -22.5 \\
\hline Right Knee Joint & 73 & 118.8 \\
\hline $\begin{array}{c}\text { Right Pitch } \\
\text { Ankle Joint }\end{array}$ & 45 & 81 \\
\hline Right Roll Ankle & 14.4 & -14.4 \\
\hline Joint & 18 & 14.4 \\
\hline Left Shoulder & -14.4 & 28.8 \\
\hline Left Elbow & -3.6 & -3.6 \\
\hline Right Shoulder & -27 & -27 \\
\hline Right Elbow & & \\
\hline
\end{tabular}

\title{
5. CONCLUSION
}

Experimental results carried out during the KHR-2HV simulation model by Webots. This model gives a better estimation to confirm the stability of the 10 (DoFs) and 17 (DoFs) bipedal robots. The walking states for 17 (DoFs) are beginning to fulfill the stability from [Right or left roll hip joint, Left or right roll hip joint, Right or left shoulder and arm, Left or right knee, Left or right pitch hip joint, Left or right pitch ankle joint, Right or left roll ankle joint] respectively. The cubic polynomial foot trajectory equations are estimated for ten DoFs and seventeen (DoFs) bipedal robots are $y=4 \times 10^{-16} z^{3}-$ $0.0433 z^{2}+0.4329 z+0.7619$ with regression 0.9276 and $y=-0.000074 z^{3}-0.13 z^{2}+$ $0.671 z+1.1326$ with regression 0.939 respectively.

\section{NOMENCLATURE}

$C=$ constant.

$D_{s}=$ the length of one step, $\mathrm{m}$.

$\mathrm{g}=$ gravitational acceleration, $\mathrm{m} / \mathrm{s}^{2}$.

$h_{o}, L_{s}=$ the values of the highest point of the trajectory of swing leg.

$F_{p}=$ external force, $\mathrm{N}$.

$f=$ foot rotation indicator, $\mathrm{cm}$.

$\mathrm{H}=$ total angular momentum.

$I=$ inertia tensor.

$M=$ moment, N.m.

$\mathrm{m}=$ mass of robot, $\mathrm{g}$.

$\mathrm{P}=$ total linear momentum.

$p=$ zero moment point, $\mathrm{cm}$.

$q_{f}$ and $q_{b}=$ the preferred swing foot slope angles as it lands and leaves on the floor. $q_{s}$ and $q_{e}=$ the slopes of the floor surface.

$\mathrm{R}=$ rotation matrix.

$R=$ reaction force of the foot, $\mathrm{N}$.

$T_{C}=$ walking step period, sec.

$x_{Z M P}=$ zero moment point in $\mathrm{x}$-axis, $\mathrm{cm}$.

$y_{Z M P}=$ zero moment point in $\mathrm{y}$-axis, $\mathrm{cm}$.

$\emptyset=$ the rotation angle.

$\omega=$ angular velocity, red/s.

\author{
ABBREVIATIONS \\ $\mathrm{CoM}=$ center of mass. \\ $\mathrm{CoP}=$ center of pressure. \\ $\mathrm{CP}=$ capture point. \\ $\mathrm{CPU}=$ central processing unit. \\ DoFs $=$ degrees of freedom. \\ $\mathrm{DSP}=$ double support phase \\ $\mathrm{FPC}=$ foot positioning compensator. \\ $\mathrm{FRI}=$ foot rotation indicator. \\ LIPM = linear inverted pendulum model. \\ $\mathrm{PIC}=$ programmable integrated circuit. \\ $\mathrm{PWM}=$ pulse width modulation . \\ $\mathrm{SL}=$ step length. \\ $\mathrm{SSP}=$ single support phase. \\ $\mathrm{ZMP}=$ zero moment point.
}

\section{ACKNOWLEDGEMENTS}

A part of this research is supported by mechanical engineering/ College of Engineering/ University of Baghdad.

\section{REFERENCES}

[1] Q. Huang et al., "High stability, smooth walking pattern for a biped robot," Proc. IEEE Int. Conf. Robot. Autom., vol. 1, no. May, pp. 65-71, 1999,

[2] S. Kajita et al., "Kajita_2010_Biped Walking Stabilization Based on Linear Inverted Pendulum Tracking,” pp. 4489-4496, 2010.

[3] P. R. Vundavilli and D. K. Pratihar, "Balanced gait generations of a two-legged robot on sloping surface," Sadhana - Acad. Proc. Eng. Sci., vol. 36, no. 4, pp. 525-550, 2011.

[4] S. Warnakulasooriya, A. Bagheri, N. Sherburn, and M. Shanmugavel, "Bipedal walking robot - A developmental design," Procedia Eng., vol. 41, pp. 1016-1021, 2012.

[5] I. S. Kim, Y. J. Han, and Y. D. Hong, "Stability Control for Dynamic Walking of Bipedal Robot with Real-time Capture Point Trajectory Optimization," J. Intell. Robot. Syst. Theory Appl., vol. 96, no. 3-4, pp. 345-361, 2019.

[6] C. Liu, T. Zhang, M. Liu, and Q. Chen, “Active Balance Control of Humanoid Locomotion 
Based on Foot Position Compensation," J. Bionic Eng., vol. 17, no. 1, pp. 134-147, 2020.

[7] A. Fawzi, A. Kareem, A. Abdul, H. Ali, and A. Kareem, "Robust Stability Control of Inverted Pendulum Model for Bipedal Walking Robot,", NJES, vol. 23, no. 1, pp. 81-88, 2020.

[8] N. Fatehi, A. Akbarimajd, and M. Asadpour, "ZMP analysis for dynamic walking of a passivity-based biped robot with flat feet," ICCAS 2010 - Int. Conf. Control. Autom. Syst., pp. 1419-1423, 2010.

[9] Y. D. Hong, "Capture point-based controller using real-time zero moment point manipulation for stable bipedal walking in human environment," Sensors (Switzerland), vol. 19, no. 15, 2019.

[10] B. P. George and A. P. Sudheer, "Dynamic biped locomotion in structured environment," ACM Int. Conf. Proceeding Ser., no. 1, pp. 16, 2013.

[11]M. H. P. Dekker, "Zero-Moment Point Method for Stable Biped Walking," Report, no. July, p. 62, 2009.

[12] A. Goswami, "Foot rotation indicator (FRI) point: a new gait planning tool to evaluate postural stability of biped robots," Proc. - IEEE Int. Conf. Robot. Autom., vol. 1, no. May, pp. 47-52, 1999.

[13]K. Erbatur, A. Okazaki, K. Obiya, T. Takahashi, and A. Kawamura, "A study on the zero moment point measurement for biped walking robots," Int. Work. Adv. Motion Control. AMC, pp. 431-436, 2002.
[14]B. Earl, “Adafruit PCA9685 16-Channel Servo Driver Using the Adafruit Library Install Adafruit PCA9685 library Test with the Example Code: Using as GPIO Arduino Library Docs Python \& CircuitPython," 2018.

[15]V. V. Madadi and S. Tosunoglu, "Design and development of a biped robot," Proc. 2007 IEEE Int. Symp. Comput. Intell. Robot. Autom. CIRA 2007, pp. 243-247, 2007.

[16]A. Formalskii and A. Shneider, Book Reviews : Biped Locomotion (Dynamics, Stability, Control and Application), vol. 11, no. 4. 1992.

[17]D. Wollherr, "Design and Control Aspects of Humanoid Walking Robots," Science (80-.)., 2005.

\section{دراسة عملية ومحاكاة لدورة المشي لنوعين من درجات الحرية للروبوت ثنائي الأرجل \\ احمد عبدالحسين علي \\ ahmedrobot65@yahoo.com \\ علي فوزي عبدالكريم \\ bdulkareem0903@coeng.uobaghdad.edu.iq}

جامعة بغداد ـ كلية الهندسة ـ قسم الهندسة الميكانيكية

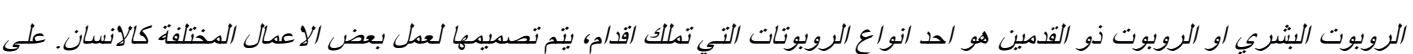

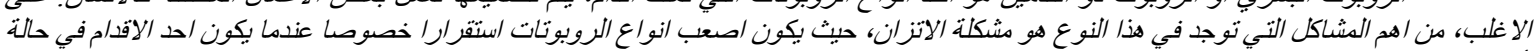

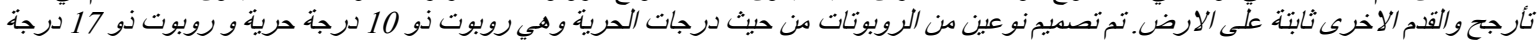

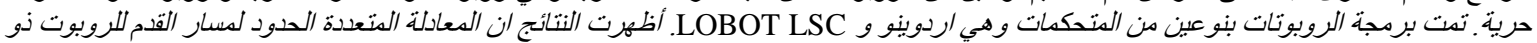

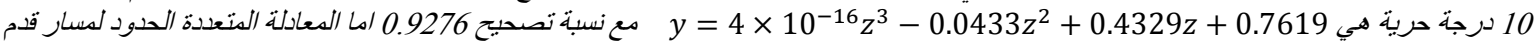

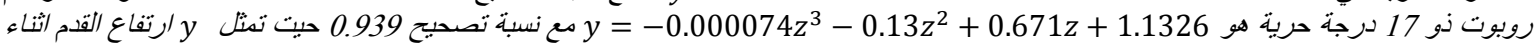

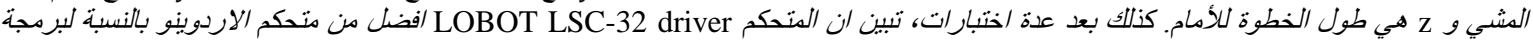

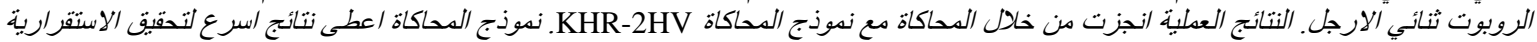

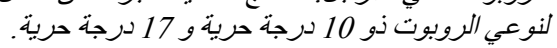

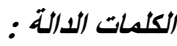

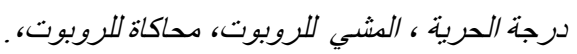

